\title{
The relationship between sexual sensation seeking and problematic Internet pornography use: A moderated mediation model examining roles of online sexual activities and the third-person effect
}

\author{
LIJUN CHEN ${ }^{1,2,3}$, YING YANG ${ }^{2}$, WENLIANG SU ${ }^{1,2,3}$, LIJUN ZHENG ${ }^{4}$, CODY DING ${ }^{5}$ and MARC N. POTENZA ${ }^{3,6,7,8,9 *}$ \\ ${ }^{1}$ Department of Psychology, Institute of Psychological and Cognitive Sciences, Fuzhou University, Fuzhou, China \\ ${ }^{2}$ Department of Psychology, School of Humanities and Social Sciences, Fuzhou University, Fuzhou, China \\ ${ }^{3}$ Department of Psychiatry, Yale University School of Medicine, New Haven, CT, USA \\ ${ }^{4}$ Faculty of Psychology, Southwest University, Chongqing, China \\ ${ }^{5}$ Educational Psychology, University of Missouri-St. Louis, St. Louis, MO, USA \\ ${ }^{6}$ Department of Neuroscience, Yale University School of Medicine, New Haven, CT, USA \\ ${ }^{7}$ Department of Child Study Center, Yale University School of Medicine, New Haven, CT, USA \\ ${ }^{8}$ Connecticut Mental Health Center, New Haven, CT, USA \\ ${ }^{9}$ Connecticut Council on Problem Gambling, Wethersfield, CT, USA
}

(Received: April 5, 2018; revised manuscript received: June 26, 2018; accepted: July 26, 2018)

\begin{abstract}
Background and aims: Internet pornography consumption is prevalent among college students and problematic for some, yet little is known regarding the psychological constructs underlying problematic Internet pornography use (PIPU). Drawing on the Interaction of Person-Affect-Cognition-Execution model, this study tested a model that sexual sensation seeking (SSS) would impact PIPU through online sexual activities (OSAs) and that this relationship would be influenced by the third-person effect (TPE; a social cognitive bias relating to perceived impacts on others as compared to oneself) in a gender-sensitive manner. Methods: A total of 808 Chinese college students (age range: 17-22 years, 57.7\% male) were recruited and surveyed. Results: Men scored higher than women on OSAs and PIPU and on each scale's component factors. The relationship between SSS and PIPU was mediated by OSAs, and the TPE moderated this relationship: the predictive path (SSS to PIPU) was significant only in participants with high TPE. The moderated mediation model was not invariant across gender groups, with data suggesting that it accounted for a greater proportion of the variance in men as compared with women. Discussion and conclusions: The findings suggest that SSS may operate through participation in OSAs to lead to PIPU, and this relationship is particularly relevant for college-aged males scoring high on the TPE. These findings have implications for individuals who might be particularly vulnerable to developing PIPU and for guiding educational efforts and targeting interventions in college-aged students. The extent to which these findings extend to other age groups and cultures warrants further examination.
\end{abstract}

Keywords: gender, moderated mediation, online sexual activities, problematic Internet pornography use, sexual sensation seeking, third-person effect

\section{INTRODUCTION}

The use of the Internet for sexual exploration is prevalent with $13 \%$ of terms entered in Internet search engines relating to sex (Ogas \& Gaddam, 2011). About $90 \%$ of adults in mainland China have been engaged in online sexual activities (OSAs) over a 6-month period (Li \& Zheng, 2017; Zheng \& Zheng, 2014). Many college students reported experience in accessing sexual information $(89.8 \%)$ and sexual entertainment (76.5\%) online, and almost half of them (48.5\%) reported browsing sexual products (Döring, Daneback, Shaughnessy, Grov, \& Byers, 2017). In most cases, pornography viewing is not associated with interference in major areas of life functioning. However, for some, it may become problematic and be associated with negative consequences (Ford, Durtschi, \& Franklin, 2012; Weaver et al., 2011). For these reasons, it is important to investigate the potential mechanisms that may contribute to the development and maintenance of problematic Internet pornography use (PIPU).

Similar to gambling disorder or other excessive online activities, dysfunctional involvement in online pornography use has been conceptualized as a "behavioral" addiction (Cooper, Delmonico, Griffin-Shelley, \& Mathy, 2004). PIPU appears to share several core features with other addictive behaviors (Brand et al., 2011). It typically involves poorly controlled and excessive use, strong desires, motivations and craving, obsessive thoughts, and continued

* Corresponding author: Marc N. Potenza; Connecticut Mental Health Center, 34 Park Street, New Haven, CT 06519, USA; Phone: +1 203974 7356; Fax: +1 203974 7366; E-mail: marc. potenza@yale.edu

This is an open-access article distributed under the terms of the Creative Commons Attribution-NonCommercial 4.0 International License, which permits unrestricted use, distribution, and reproduction in any medium for non-commercial purposes, provided the original author and source are credited, a link to the CC License is provided, and changes - if any - are indicated. 
engagement despite adverse consequences, which, in turn, lead to significant personal distress and functional impairment (Cooper et al., 2004; Kor et al., 2014; Wéry \& Billieux, 2015). PIPU may have elements related to both problematic Internet use (PIU) and sexual addiction (Griffiths, 2012) or compulsive sexual behavior disorder (Kraus et al., 2018), perhaps as a specific subtype of each (Brand, Young, \& Laier, 2014; Young, 2008).

As a potential personality trait, sexual sensation seeking (SSS) has been suggested to lead to PIPU (Perry, Accordino, $\&$ Hewes, 2007). SSS refers to tendencies to try unique and novel sexual experiences to achieve high degrees of sexual arousal (Kalichman et al., 1994). It has been associated with hypersexuality (Walton, Cantor, Bhullar, \& Lykins, 2017), risky sexual behaviors (Heidinger, Gorgens, \& Morgenstern, 2015), and high frequency of OSAs (Lu, Ma, Lee, Hou, \& Liao, 2014; Luder et al., 2011; Peter \& Valkenburg, 2011; Zheng, Zhang, \& Feng, 2017; Zheng \& Zheng, 2014). Hence, SSS is an important variable to study the development of PIPU. Yet, the mechanisms by which SSS may lead to PIPU remain unclear. An improved understanding of such mechanisms could facilitate practical guidance to individuals and healthcare practitioners and educators on how to develop intervention strategies (MacKinnon \& Luecken, 2008). To understand the implications of SSS for PIPU completely, research should investigate the potential causal pathways that are involved in the process by which SSS-related impacts unfold (i.e., mediation). Second, the models should illuminate contextual factors on which SSS-related impacts depend (i.e., moderation). Consequently, this study investigates relationships between SSS and other factors that may explain mechanisms by which SSS may lead to PIPU (mediation) and factors that may influence these pathways (moderation).

To understand relationships between SSS and PIPU in more detail, we draw on the Interaction of Person-AffectCognition-Execution (I-PACE) model of specific Internetuse disorders (Brand et al., 2014; Brand, Young, Laier, Wölfling, \& Potenza, 2016). The model posits that the development and maintenance of specific forms of PIU may be driven by a person's core characteristics (needs, goals, specific predispositions, and psychopathology) and influenced by a person's cognitions, and these may lead to specific behaviors such as OSAs. If an individual achieves gratification from the behavioral engagement, it may become reinforced and repeated; in the case of OSAs, this may then lead to PIPU, consistent with existing data relating sexual satisfaction, cybersex, and PIPU (Lu et al., 2014). Addiction models also posit that sensation-seeking tendencies linked to positive reinforcement motivations are related to addictive behaviors (Steinberg et al., 2008). As the Internet contains many opportunities for engaging in OSAs (relating to pornography viewing, sharing of sexually explicit materials, etc.), individuals with higher SSS, who are looking for novel sexual stimuli, may be particularly vulnerable to developing PIPU through their engagement in OSAs. The I-PACE model provides a theoretical explanation for the impact of SSS on PIPU. Consistent with this notion, the relationship between the frequencies of OSAs and PIPU has been observed to be positive (Twohig, Crosby, \& Cox, 2009). Other researchers have proposed that the frequency of pornography use is an important consideration in PIPU development (Cooper, Delmonico, \& Burg, 2000; Cooper et al., 2004), but it is not the sole criterion for problematic use, particularly if the behavior is controlled in the setting of other priorities and not leading to harm or distress (Bőthe et al., 2017; Kor et al., 2014; Wéry \& Billieux, 2015).

The I-PACE model considers that the Internet-related cognitive biases may include false beliefs about the possible effects of using certain applications/sites. The perception that media exerts a stronger effect on others than on oneself has been termed the third-person effect (TPE) and perceiving influences on oneself to be greater than those on others has been called the first-person effect (Davison, 1983). According to the I-PACE model, cognitive biases may act in concert with users' predisposing characteristics, accelerate the intensities of cue-reactivity and desire, and promote the use of special applications/sites (Brand, Young, et al., 2016). With particular relevance to this study, people who report higher levels of SSS have been reported to be more individualistic (Gaither \& Sellbom, 2003), and the individualism may promote the belief that Internet pornography may have more negative influences on others than on themselves (Lee \& Tamborini, 2005). The TPE may lead to a lower awareness regarding negative consequence of Internet pornography and thus may be positively linked to the development of PIPU.

In this theoretical framework, we sought to examine whether the impact of SSS on PIPU is mediated by OSAs and whether this relationship is moderated by the levels of the TPE. Therefore, we constructed a moderated mediation model (Figure 1). Given that significant gender-related differences exist in OSAs and PIPU (Kor et al., 2014; Turban, Potenza, Hoff, Martino, \& Kraus, 2017), and male engagement in potentially addictive behaviors is linked more strongly to positive-reinforcement motivations than female engagement (Potenza et al., 2012; Zakiniaeiz, Cosgrove, Mazure, \& Potenza, 2017), we examined the extent to which the model was sensitive to gender.

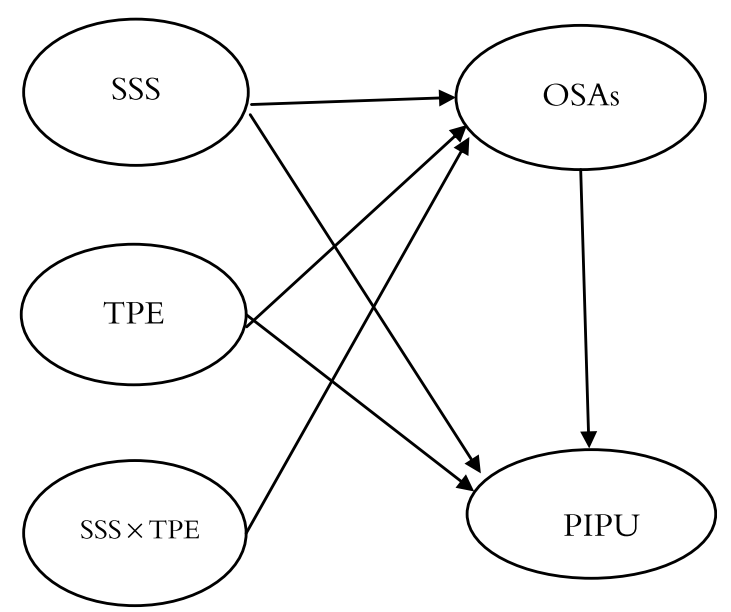

Figure 1. Hypothesis model. SSS: sexual sensation seeking; TPE: third-person effect; PIPU: problematic Internet pornography use; OSA: online sexual activities 


\section{METHODS}

\section{Participants and procedures}

The data were collected in the period between November 2016 and March 2017 from a sample of Chinese college students through an online-based survey. A total of 808 college students [ 466 men, 342 women; $M_{\text {age }}=18.54$ years, standard deviation $(S D)=0.75]$ were recruited through the Chinese professional survey website (www.sojump.com). No monetary incentives were provided for participation. The volunteers were from key public universities $(n=276)$, first-tier universities $(n=200)$, second-tier universities $(n=150)$, community colleges, and vocational colleges $(n=182)$. Anonymity of the participants was protected (no personal data or Internet protocol addresses were collected).

\section{Outcome measures}

The PIPU Scale (PIPUS) is a 12-item self-report scale that is based on the Problematic Pornography Use Scale (Kor et al., 2014) and was used to assess PIPU. The scale consists of four factors including (a) distress and functional problems, (b) excessive use, (c) self-control difficulties, and (d) use in order to escape or avoid negative emotions. In this study, "pornography" was modified into "Internet pornography" from the original scale. Each factor of the PIPUS includes three items. Respondents were asked to report on their Internet pornography use in the last 6 months on a 6-point Likert scale ranging from 0 (never) to 5 (all the time) with higher scores reflecting greater PIPU severity. Cronbach's $\alpha$ values for these four factors and the total score in this study were $.78, .85, .90, .87$, and .94 . The Chinese version of the scale has been found to be reliable and valid among Chinese college students (Chen, Wang, Chen, Jiang, \& Wang, 2018).

OSAs were assessed using 13 items from a scale that measured participants' use of the Internet for (a) viewing sexually explicit material, (b) seeking sexual partners, (c) cybersex, and (d) flirting and relationship maintenance (Zheng \& Zheng, 2014). Assessed items were rated from 1 (never) to 9 (at least once a day). Higher scores reflected more frequent engagement in OSAs. The viewing of sexually explicit material section included five items about visiting erotic websites, viewing and downloading erotic videos online, and reading erotic material online (Cronbach's $\alpha=.86$ ). There were two items that measured the frequency of seeking sexual partners, including the number of sexual partners sought and the number of sexual partners found online (Cronbach's $\alpha=.70$ ). Frequency of cybersex was assessed using four items including masturbating or viewing strangers masturbating via a webcam, describing real-time sexual fantasies via typing or voice, and exchanging erotic pictures online (Cronbach's $\alpha=.80$ ). Flirting and sexual relationship maintenance were measured using two items (Cronbach's $\alpha=.64$ ). Cronbach's $\alpha$ of the whole scale was .89 .

The TPE was measured by asking two separate questions: "How much influence does Internet pornography have on you/other students at your university? (e.g., influence on your/others' sexual morality and attitudes toward the opposite sex)," according to the definition of Davison (1983), Lo, Wei, and $\mathrm{Wu}$ (2010), and Zhao and Cai (2008). Participants answered these questions on a 7-point scale, from 1 (no influence at all) to 7 (a great deal of influence). TPE scores were derived by subtracting perceived influence on self from perceived influence on other students with greater than 0 representing a TPE and less than 0 representing a first-person effect (Golan \& Day, 2008). In order to reduce test cross-reference influences, the two items were imbedded in the two parts of the questionnaire.

The Sexual Sensation Seeking Scale (SSSS) was developed by Kalichman et al. (1994) to measure the degree of SSS. The SSSS is an 11-item Likert-type measure with response options ranging from 1 (not at all like me) to 4 (very much like me). The 11-item scale includes statements such as, "I am interested in trying out new sexual experiences" and "I feel like exploring my sexuality." Higher scores reflect stronger SSS tendencies. The internal consistency (Cronbach's $\alpha$ ) of the SSSS was .92 .

\section{Statistical analysis}

Gender-related differences in clinical characteristics were examined using one-way multivariate analyses of variance (MANOVAs). Controlling for age and gender and partial correlation analyses were employed to measure the strengths of relationships between the main research variables including PIPU, OSAs, SSS, and TPE. Mplus7.2 was used to assess the moderated mediation model of SSS and PIPU and the potential variance of the model by gender. We estimated the significance of standardized coefficients with 1,000 bootstrap iterations. In this study, standard errors and confidence intervals of parameter estimations were obtained. If the $95 \%$ confidence intervals did not contain zero, the findings were considered statistically significant.

\section{Ethics}

The study protocol and materials were approved by the ethical committee of the Institute of Psychological and Cognitive Sciences, Fuzhou University, China. All subjects were informed about the study and all provided informed consent.

\section{RESULTS}

\section{Descriptive statistics for the variables}

Among the whole sample, mean score was 7.13 for PIPU $(S D=8.48$, skewness $=1.97$, and kurtosis $=5.55)$ and for frequency of OSAs $1.70(S D=0.94$, skewness $=2.84$, and kurtosis $=12.34$ ). Men had higher scores on the PIPUS and engaged in OSAs more frequently when compared to women (Table 1). Further analysis [one-way MANOVA with the scores of the four OSA subscales, multivariate $F(4,803)=26.12, p<.001$, partial $\eta^{2}=0.12$, and the four PIPUS sub-scales, multivariate $F(4,803)=12.91, p<.001$, partial $\eta^{2}=0.06$, respectively] demonstrated that this pattern extended to each scale's component factors. 


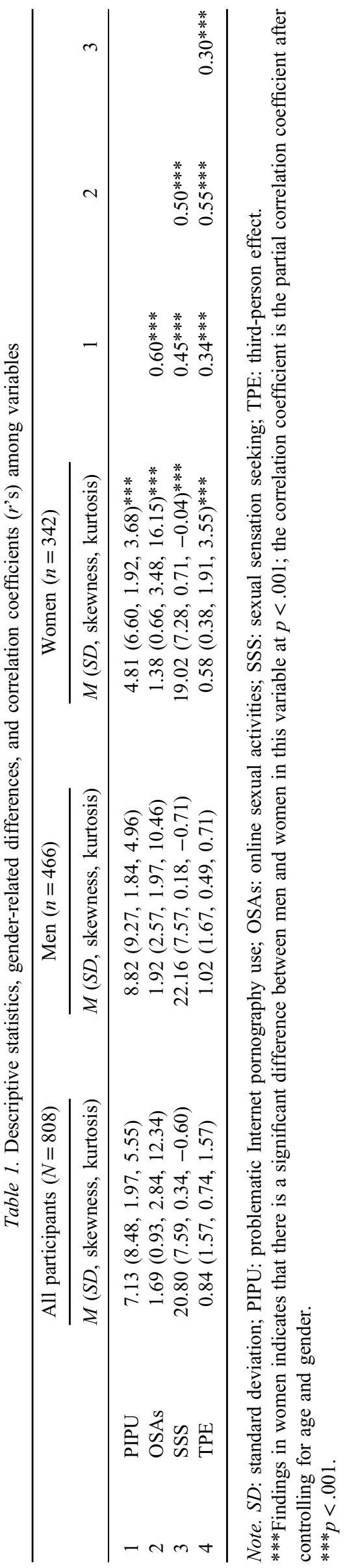

Relationships between SSS, TPE, OSAs, and PIPU

Pearson's partial correlation coefficients between PIPU, OSAs, SSS, and TPE are shown, controlling for age and gender (Table 1). PIPU total score and its factors were highly correlated with OSAs. As might be expected, correlations with PIPU scores were typically numerically most robust for viewing sexual explicit material $(r=.65$, $p<.001)$ and least robust for flirting and relationship measures $(r=.21, p<.001)$. Both SSS and TPE were positively correlated with OSAs and PIPU measures and with each other. The results suggest that with greater SSS, there was increased likelihood of engagement in OSAs and use of Internet pornography problematically.

The influence of SSS on PIPU: OSAs as a mediating factor and TPE as a moderator

According to Edwards and Lambert (2007), it is necessary to test the parameters of the three regression equations in the intermediate model with moderated front paths: (a) Equation 1 tests the regulating effect of the variable (TPE represented by $\mathrm{U}$ ) on the independent variable (SSS represented by X) and the dependent variable (PIPU represented by Y). (b) Equation 2 estimates the regulatory role of the moderating variable (TPE) on the independent variable (SSS) and intermediary variables (OSA represented by W). (c) Equation 3 tests the moderating effect of the moderator (TPE) on the relationship between the independent variable (SSS) and intermediary variable (OSA), and mediating effects of the intermediary variable (OSA) on the dependent variable (PIPU represented by Y). The values of SSS and TPE were $z$-standardized to $z$-scores, and then these two $z$-scores were multiplied as points of interaction (Dawson, 2014).

As shown in Table 2, in Equation 1, the interaction effect of SSS and TPE significantly predicted PIPU $\left(c_{3}=0.42\right)$ and hence the further analyses were conducted. In Equation 2, the path of the interaction of SSS and TPE was significant $\left(a_{3}=0.37\right)$. In Equation 3, the path from OSAs to PIPU was significant $\left(b_{1}=0.56\right)$, and both $a_{3}$ and $b_{1}$ were significant. In addition, $a_{1}$ and $b_{2}$ and $a_{3}$ and $b_{2}$ were all significant, which met the testing criteria (Edwards \& Lambert, 2007). At the same time, as shown in Table 2, in Equation 3, the moderating model had increased $8.9 \%$ variable explanatory volume compared with Equation 1, which supported the mediating role of OSAs in the impact of SSS on problematic use. The hypothesized model in Figure 1 was thus tested and supported.

From Table 2, the positive coefficient of the interaction term suggested that it became more positive as the TPE increased. To enable easier interpretation, we plotted the relationship so it may be viewed visually. We divided the individuals' scores into high and low groups in accordance with a $S D$ above and below the mean (Dawson, 2014). The results showed that for the participants with high TPE (with scores a $S D$ above the mean), SSS could positively predict OSAs $(\beta=0.71, t=6.13, p<.01)$, whereas for the participants with low scores on the TPE (with the scores of a $S D$ under the mean), the predictive effect of SSS was not significant $(\beta=-0.04, t=0.27, p=.79$; Figure 2$)$. 


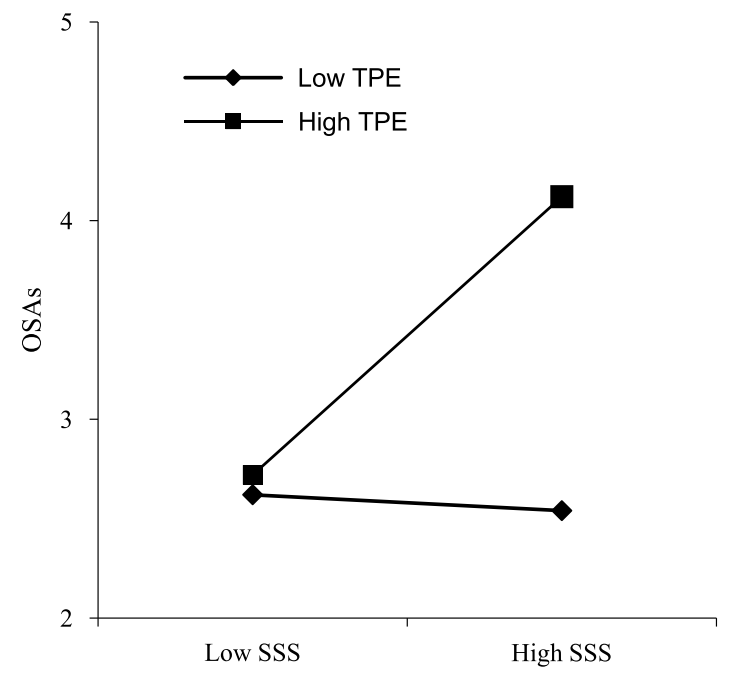

Figure 2. Relationships between SSS, TPE, and OSAs

\section{Multiple group invariance test of the model in men and women}

Measurement invariance was employed to examine the moderated mediation model across the two genders. Multigroup invariant tests usually require several steps, including the gradual addition of constraints (qualifying additional parameters to be equal) in each step to complete the overall testing, using the fit indicator difference as criteria to test whether the equal assumption hurts the performance of model fitting. If the fitting indices are not ideal, it is stated that there is no equivalent structure between the two groups and the test is stopped (Lomazzi, 2018). The first step is the configural invariance test (the baseline model) where no parameters are set for cross-group equality to see if the model "looks" the same in both groups. In this step, the results showed that the suitability indexes of the model were: $\chi^{2}=703.11, d f=77, p<.001$, comparative fit index $(\mathrm{CFI})=0.86$, Tucker-Lewis index $(\mathrm{TLI})=0.81$, and root mean square error of approximation $($ RMSEA $)=0.14$. It illustrated that the baseline model was rejected, which meant that at least the structural equation model of one group should be modified. In step zero, the baseline models were also estimated for both males and females, showing a good fit in male subjects $\left(\chi^{2}=101.72, d f=29, p<.001\right.$, $\mathrm{CFI}=0.97, \mathrm{TLI}=0.95$, and $\mathrm{RMSEA}=0.073$ ) but not in female subjects $\left(\chi^{2}=216.256, d f=29, \quad p<.001\right.$, $\mathrm{CFI}=0.90$, TLI $=0.82$, and RMSEA $=0.14$ ). This finding suggested that the moderated mediation model depend on gender. The variation interpretation of the model was $57.5 \%$ among men and 32.5\% among women (Table 3 ).

\section{DISCUSSION}

In this study, we tested relationships between TPE, SSS, OSAs, and PIPU in young adult college-attending men and women from China. Specifically, we tested a moderated mediation model embedded within the I-PACE theoretical framework within the context of the PIU and addiction 
Table 3. Comparison of path coefficients of the moderated mediation model between men and women

\begin{tabular}{|c|c|c|c|c|c|c|c|}
\hline \multirow[b]{2}{*}{ Independent variables } & \multirow[b]{2}{*}{ Dependent variables } & \multicolumn{4}{|c|}{ Path coefficient } & \multirow[b]{2}{*}{ Critical ratios } & \multirow[b]{2}{*}{$p$} \\
\hline & & $\operatorname{Men}(\beta)$ & $S E$ & Women $(\beta)$ & $S E$ & & \\
\hline SSS & OSAs & 0.39 & 0.05 & 0.40 & 0.06 & 1.89 & .059 \\
\hline TPE & & 0.45 & 0.05 & 0.47 & 0.07 & -6.85 & .000 \\
\hline $\mathrm{SSS} \times \mathrm{TPE}$ & & 0.33 & 0.08 & 0.60 & 0.07 & -27.10 & .000 \\
\hline SSS & PIPU & 0.17 & 0.07 & 0.03 & 0.06 & 14.89 & .000 \\
\hline TPE & & 0.09 & 0.03 & 0.13 & 0.03 & 10.75 & .000 \\
\hline $\mathrm{SSS} \times \mathrm{TPE}$ & & 0.004 & 0.06 & 0.24 & 0.08 & 14.38 & .000 \\
\hline OSAs & & 0.74 & 0.11 & 0.89 & 0.07 & -1.95 & .258 \\
\hline
\end{tabular}

Note. The $\beta$ is the standardized path coefficient. The $p$ values of ".000" correspond to $p<.001$. SSS: sexual sensation seeking; PIPU: problematic Internet pornography use; OSAs: online sexual activities; TPE: third-person effect; $S E$ : standard error.

literatures. Previous research has examined the influences of negative reinforcement (i.e., escape or avoidance of depression and anxiety through pornography viewing) on the development of PIPU (Paul \& Shim, 2008). While several studies have also investigated the aspects of positive reinforcement and SSS as related to PIPU (Steinberg et al., 2008), mechanistic explanations for relationships between SSS and PIPU have been largely lacking. To advance knowledge in this area and offer more specific guidance to individuals, therapists and educators, this study demonstrates that SSS exerts its influences on PIPU through OSAs, is moderated by the TPE, and seems to be mainly applicable to males. Specifically, we found that SSS leads to more frequent OSAs typically when individuals perceive influence on others as greater than those on themselves, which provides more detailed and specific explanations of when, or under what conditions, SSS may lead to more OSAs and subsequently PIPU. Furthermore, this pathway accounted for greater variance among men than women. Thus, our hypotheses were largely supported and the implications are listed below.

The moderated mediation model indicates that the relationship between higher SSS and PIPU operates through more frequent OSAs, consistent with prior findings (Hong et al., 2012; Zheng \& Zheng, 2014). Sensation seeking is a tendency frequently associated with addictive behaviors (Steinberg et al., 2008). As an important component of sensation seeking, SSS reflects a tendency to seek risky, exciting, and novel sexual experiences. The findings are consistent with the notion that the Internet may attract particular users of pornography, especially male college students high in SSS because of its novelty, anonymity, low cost, and easy access (Cooper et al., 2000). For individuals with high SSS, the Internet may represent a new way to satisfy the desires of seeking novel sexual stimulation, get pleasure, and relieve pain related to the suppression of sexual impulses, then generate a conditioned behavioral pattern (Putnam, 2000) in consistent with the I-PACE model (Brand, Young, et al., 2016). Nonetheless, such a conditioned relationship could make it increasingly hard for an individual to control one's use of Internet pornography, even though negative consequences related to overuse may be occurring. Furthermore, it has been suggested that users, perhaps those high in SSS, who have certain sexual fantasies that may be satisfied by Internet pornography better than by real-world sex may be at particular risk for developing PIPU
(Brand, Snagowski, Laier, \& Maderwald, 2016; Cooper et al., 2000, 2004). These possibilities warrant direct examination in future studies, for example, by including measures of sexual expectancies in longitudinal studies of Internet pornography use.

The current findings indicate that individuals who believe that Internet pornography may have damaging effects more on others than on themselves were more likely to engage in OSAs and thus experience PIPU. According to Perloff's (2002) "self-expanding" mechanism, people may tend to highlight a "super-self-image" both internally and externally to protect and improve "the self." The extent to which this theory may explain tendencies to potentially deny or underestimate the effects of pornography consumption and how this may relate to developing PIPU warrant additional research (Sun, Pan, \& Shen, 2008). Furthermore, as individuals with high SSS may have greater individualistic tendencies, their characteristics may lead them to pay more attention to their own experiences, and this may strengthen the self-other cognitive bias on the influence of Internet pornography, thus promoting beliefs that they may not be negatively affected (Lee \& Tamborini, 2005).

Multigroup invariance testing showed that the model tested in this study did not apply equally across genders with the model applying to males more so than females. First, males tend to pursue more varied sexual experiences (Oshri, Tubman, Morganlopez, Saavedra, \& Csizmadia, 2013); they also report more sexual arousal (Goodson, McCormick, \& Evans, 2000), sexual excitement, and masturbation when browsing pornographic materials online with females reporting more avoidance, disgust, or worries (González-Ortega \& Orgaz-Baz, 2013). Therefore, males, particularly those high in SSS, may be more likely to seek novel sexual stimulation online that may lead to PIPU. Second, emotional factors may be more important in relation to sexual behaviors for females when compared to males (Cooper, Morahan-Martin, Mathy, \& Maheu, 2002), but most sexual explicit materials available online may be less desirable for females (Gonzalez-Ortega \& Orgaz-Baz, 2013). Finally, women also in general view pornography more negatively than men (Malamuth, 1996). Therefore, in this multiple model, the functions of SSS and the TPE may vary for multiple reasons, and these possible reasons should be examined in future studies.

Our findings have potential implications for sexual education, prevention of PIPU, and Internet media regulation. 
First, the study found that SSS may predict OSAs and PIPU, particularly in men who have high SSS. SSS should be examined further and in educational contexts, it may be particularly important to instruct individuals as how best to understand and meet their own sexual needs in healthy fashions. Such efforts may occur best prior to college in sex education during adolescent or earlier periods, given that the proportion of youth as young as 10 years of age who views pornography may be rising (Peter \& Valkenburg, 2016). Second, the influence of the TPE suggests that education regarding the potentially harmful effects of Internet pornography may help reduce the likelihood of developing PIPU. Such educational efforts may involve education about young men who report that Internet pornography viewing has altered their sexual arousal templates and led to erectile dysfunction (Wéry \& Billieux, 2016). Finally, the psychological mechanisms of relating to male and female engagement in OSAs and development of PIPU may be different. Therefore, educational guidance and evaluation efforts may need to be tailored in gender-sensitive fashions, with particular emphasis on SSS in males and perhaps other domains (e.g., relating to emotional and social aspects) being more relevant to females, although this latter point is currently speculative based on the recent findings.

Our results should be considered in light of study limitations. The survey did not employ random sampling and was Internet-based, and these features could limit the generalizability of the findings. Second, participants were college students from China. While pornography consumption is very relevant to this group, the extent to which the findings may generalize to other young adults and age groups, groups with less education, and other jurisdictions and cultures warrants additional investigation. Third, as other factors have been linked to OSAs and PIPU, such as low selfesteem (Kor et al., 2014), craving (Kraus, Martino, \& Potenza, 2016), and poor emotion-regulation strategies (Wéry \& Billieux, 2015), these should be examined in future studies. Despite these limitations, the study provides important data supporting and refining proposed theoretical models for specific forms of PIU and suggests important avenues for educational and interventional strategies to reduce the prevalence of harms associated with PIPU.

Funding sources: This work was supported by the National Social Science Foundation of China (grant number: CEA150173). Dr. MNP's involvement was supported by the National Center for Responsible Gaming. The funding agencies did not have input into the content of the manuscript and the views described in the manuscript reflect those of the authors and not necessarily those of the funding agencies.

Authors' contribution: LC designed the study, analyzed the data, and wrote the first draft of the manuscript. MNP consulted on design of the study analyses and reviewed/ revised the manuscript. YY and WS collected the data, contributed to data analyses, and reviewed/revised the manuscript. $\mathrm{CD}$ and $\mathrm{LZ}$ monitored data collection and reviewed/revised the manuscript. The authors had full access to all data in the study and take responsibility for the integrity of the data and the accuracy of the data analyses.

Conflict of interest: The authors report no conflict of interest with respect to the content of this manuscript. Dr. MNP has consulted for and advised Rivermend Health, Opiant/ Lightlake Therapeutics, and Jazz Pharmaceuticals; received research support (to Yale) from the Mohegan Sun Casino and the National Center for Responsible Gaming; consulted for legal and gambling entities on issues related to impulse control and addictive behaviors; provided clinical care related to impulse control and addictive behaviors; performed grant reviews; edited journals/journal sections; given academic lectures in grand rounds, CME events, and other clinical/scientific venues; and generated books or chapters for publishers of mental health texts. The other authors report no financial relationships with commercial interests.

\section{REFERENCES}

Brand, M., Laier, C., Pawlikowski, M., Schächtle, U., Schöler, T., \& Altstötter-Gleich, C. (2011). Watching pornographic pictures on the Internet: Role of sexual arousal ratings and psychologicalpsychiatric symptoms for using Internet sex sites excessively. Cyberpsychology, Behavior, and Social Networking, 14(6), 371-377. doi:10.1089/cyber.2010.0222

Brand, M., Snagowski, J., Laier, C., \& Maderwald, S. (2016). Ventral striatum activity when watching preferred pornographic pictures is correlated with symptoms of Internet pornography addiction. Neuroimage, 129, 224-232. doi:10.1016/j.neuroimage. 2016.01.033

Brand, M., Young, K. S., \& Laier, C. (2014). Prefrontal control and Internet addiction: A theoretical model and review of neuropsychological and neuroimaging findings. Frontiers in Human Neuroscience, 8, 375. doi:10.3389/fnhum.2014.00375

Brand, M., Young, K. S., Laier, C., Wölfling, K., \& Potenza, M. N. (2016). Integrating psychological and neurobiological considerations regarding the development and maintenance of specific Internet-use disorders: An Interaction of PersonAffect-Cognition-Execution (I-PACE) model. Neuroscience \& Biobehavioral Reviews, 71, 252-266. doi:10.1016/j. neubiorev.2016.08.033

Bôthe, B., Tóth-Király, I., Zsila, Á., Demetrovics, Z., Griffiths, M. D., \& Orosz, G. (2017). The development of the Problematic Pornography Consumption Scale (PPCS). The Journal of Sex Research, 55(3), 1-12. doi:10.1080/00224499. 2017.1291798

Chen, L. J., Wang, X., Chen, S. M., Jiang, C. H., \& Wang, J. X. (2018). Reliability and validity of the Problematic Internet Pornography Use Scale in Chinese college students. The Journal of Chinese Public Health, 34(7), 1034-1038.

Cooper, A., Delmonico, D., \& Burg, R. (2000). Cybersex users, abusers, and compulsives: New findings and implications. Sexual Addiction \& Compulsivity, 7(1-2), 5-29. doi:10.1080/1072016 0008400205

Cooper, A., Delmonico, D. L., Griffin-Shelley, E., \& Mathy, R. (2004). Online sexual activity: An examination of potentially 
problematic behaviors. Sexual Addiction \& Compulsivity, 11(3), 129-143. doi:10.1080/10720160490882642

Cooper, A. L., Morahan-Martin, J., Mathy, R. M., \& Maheu, M. (2002). Toward an increased understanding of user demographics in online sexual activities. Journal of Sex \& Marital Therapy, 28(2), 105-129. doi:10.1080/00926230252851861

Davison, W. P. (1983). The third-person effect in communication. The Public Opinion Quarterly, 47(1), 1-15. doi:10.1086/ 268763

Dawson, J. F. (2014). Moderation in management research: What, why, when and how. Journal of Business and Psychology, 29(1), 1-19. doi:10.1007/s10869-013-9308-7

Döring, N., Daneback, K., Shaughnessy, K., Grov, C., \& Byers, E. S. (2017). Online sexual activity experiences among college students: A four-country comparison. Archives of Sexual Behavior, 46(6), 1641-1652. doi:10.1007/s10508-015-0656-4

Edwards, J. R., \& Lambert, L. S. (2007). Methods for integrating moderation and mediation: A general analytical framework using moderated path analysis. Psychological Methods, 12(1), 1-22. doi:10.1037/1082-989X.12.1.1

Ford, J. J., Durtschi, J. A., \& Franklin, D. L. (2012). Structural therapy with a couple battling pornography addiction. The American Journal of Family Therapy, 40(4), 336-348. doi:10.1080/01926187.2012.685003

Gaither, G. A., \& Sellbom, M. (2003). Sexual Sensation Seeking Scale: Reliability and validity within a heterosexual college student sample. Journal of Personality Assessment, 81(2), 157-167. doi:10.1207/S15327752JPA8102_07

Golan, G. J., \& Day, G. A. (2008). The first-person effect and its behavioral consequences: A new trend in the twenty-five year history of third-person effect research. Mass Communication and Society, 11(4), 539-556. doi:10.1080/152054308023 68621

González-Ortega, E., \& Orgaz-Baz, B. (2013). Minors' exposure to online pornography: Prevalence, motivations, contents and effects. Anales De Psicología, 29(2), 319-327. doi:10.6018/ analesps.29.2.131381

Goodson, P., McCormick, D., \& Evans, A. (2000). Sex on the Internet: College students' emotional arousal when viewing sexually explicit materials on-line. Journal of Sex Education and Therapy, 25(4), 252-260. doi:10.1080/01614576.2000.11074358

Griffiths, M. D. (2012). Internet sex addiction: A review of empirical research. Addiction Research \& Theory, 20(2), 111-124. doi:10.3109/16066359.2011.588351

Heidinger, B., Gorgens, K., \& Morgenstern, J. (2015). The effects of sexual sensation seeking and alcohol use on risky sexual behavior among men who have sex with men. AIDS and Behavior, 19(3), 431-439. doi:10.1007/s10461-014-0871-3

Hong, V. N., Koo, K. H., Davis, K. C., Otto, J. M., Hendershot, C. S., \& Schacht, R. L., George, W. H., Heiman, J. R., \& Norris, J. (2012). Risky sex: Interactions among ethnicity, sexual sensation seeking, sexual inhibition, and sexual excitation. Archives of Sexual Behavior, 41(5), 1231-1239. doi:10. 1007/s10508-012-9904-Z

Kalichman, S. C., Johnson, J. R., Adair, V., Rompa, D., Multhauf, K., \& Kelly, J. A. (1994). Sexual sensation seeking: Scale development and predicting aids-risk behavior among homosexually active men. Journal of Personality Assessment, 62(3), 385-397. doi:10.1207/s15327752jpa6203_1

Kor, A., Zilcha-Mano, S., Fogel, Y. A., Mikulincer, M., Reid, R. C., \& Potenza, M. N. (2014). Psychometric development of the Problematic Pornography Use Scale. Addictive Behaviors, 39(5), 861-868. doi:10.1016/j.addbeh.2014.01.027

Kraus, S. W., Krueger, R. B., Briken, P., First, M. B., Stein, D. J., Kaplan, M. S., Voon, V., Abdo, C. H. N., Grant, J. E., Atalla, E., \& Reed, G. M. (2018). Compulsive sexual behaviour disorder in the ICD-11. World Psychiatry, 17(1), 109-110. doi:10.1002/wps.20499

Kraus, S. W., Martino, S., \& Potenza, M. N. (2016). Clinical characteristics of men interested in seeking treatment for use of pornography. Journal of Behavioral Addictions, 5(2), 169-178. doi:10.1556/2006.5.2016.036

Lee, B., \& Tamborini, R. (2005). Third-person effect and Internet pornography: The influence of collectivism and Internet self-efficacy. Journal of Communication, 55(2), 292-310. doi:10.1111/j.1460-2466.2005.tb02673.x

Li, D., \& Zheng, L. (2017). Relationship quality predicts online sexual activities among Chinese heterosexual men and women in committed relationships. Computers in Human Behavior, 70, 244-250. doi:10.1016/j.chb.2016.12.075

Lo, V. H., Wei, R., \& Wu, H. (2010). Examining the first, second and third-person effects of Internet pornography on Taiwanese adolescents: Implications for the restriction of pornography. Asian Journal of Communication, 20(1), 90-103. doi:10.1080/ 01292980903440855

Lomazzi, V. (2018). Using alignment optimization to test the measurement invariance of gender role attitudes in 59 countries. Methods, Data, Analyses (mda), 12(1), 77-103. doi:10. 12758/mda.2017.09

Lu, H., Ma, L., Lee, T., Hou, H., \& Liao, H. (2014). The link of sexual sensation seeking to acceptance of cybersex, multiple sexual partners, and one-night stands among Taiwanese college students. Journal of Nursing Research, 22(3), 208-215. doi:10.1097/jnr.0000000000000043

Luder, M. T., Pittet, I., Berchtold, A., Akré, C., Michaud, P. A., \& Surís, J. C. (2011). Associations between online pornography and sexual behavior among adolescents: Myth or reality? Archives of Sexual Behavior, 40(5), 1027-1035. doi:10. 1007/s10508-010-9714-0

MacKinnon, D. P., \& Luecken, L. J. (2008). How and for whom? Mediation and moderation in health psychology. Health Psychology, 27(2S), S99. doi:10.1037/0278-6133. 27.2(Suppl.).S99

Malamuth, N. M. (1996). Sexually explicit media, gender differences, and evolutionary theory. Journal of Communication, 46(3), 8-31. doi:10.1111/j.1460-2466.1996.tb01486.x

Ogas, O., \& Gaddam, S. (2011). A billion wicked thoughts. New York, NY: Penguin

Oshri, A., Tubman, J. G., Morganlopez, A. A., Saavedra, L. M., \& Csizmadia, A. (2013). Sexual sensation seeking, co-occurring sex and alcohol use, and sexual risk behavior among adolescents in treatment for substance use problems. The American Journal on Addictions, 22(3), 197-205. doi:10.1111/j.15210391.2012.12027.x

Paul, B., \& Shim, J. W. (2008). Gender, sexual affect, and motivations for Internet pornography use. International Journal of Sexual Health, 20(3), 187-199. doi:10.1080/1931761 0802240154

Perloff, R. M. (2002). The third-person effect. In J. Bryant \& D. Zillmann (Eds.), Media effects: Advances in theory and research (2nd ed., pp. 489-506). Mahwah, NJ: Lawrence Erlbaum Associates. 
Perry, M., Accordino, M. P., \& Hewes, R. L. (2007). An investigation of Internet use, sexual and nonsexual sensation seeking, and sexual compulsivity among college students. Sexual $A d$ diction \& Compulsivity, 14(4), 321-335. doi:10.1080/1072 0160701719304

Peter, J., \& Valkenburg, P. M. (2011). The use of sexually explicit Internet material and its antecedents: A longitudinal comparison of adolescents and adults. Archives of Sexual Behavior, 40(5), 1015-1025. doi:10.1007/s10508-010-9644-x

Peter, J., \& Valkenburg, P. M. (2016). Adolescents and pornography: A review of 20 years of research. The Journal of Sex Research, 53(4-5), 509-531. doi:10.1080/00224499.2016.1143441

Potenza, M. N., Hong, K. A., Lacadie, C. M., Fulbright, R. K., Tuit, K. L., \& Sinha, R. (2012). Neural correlates of stressinduced and cue-induced craving: Influences of gender and cocaine dependence. American Journal of Psychiatry, 169(4), 406-414. doi:10.1176/appi.ajp.2011.11020289

Putnam, D. E. (2000). Initiation and maintenance of online sexual compulsivity: Implications for assessment and treatment. CyberPsychology \& Behavior, 3(4), 553-563. doi:10.1089/ 109493100420160

Steinberg, L., Albert, D., Cauffman, E., Banich, M., Graham, S., \& Woolard, J. (2008). Age differences in sensation seeking and impulsivity as indexed by behavior and self-report: Evidence for a dual systems model. Developmental Psychology, 44(6), 1764-1778. doi:10.1037/a0012955

Sun, Y., Pan, Z., \& Shen, L. (2008). Understanding the thirdperson perception: Evidence from a meta-analysis. Journal of Communication, 58(2), 280-300. doi:10.1111/j.1460-2466. 2008.00385.x

Turban, J. R., Potenza, M. N., Hoff, R. A., Martino, S., \& Kraus, S. W. (2017). Psychiatric disorders, suicidal ideation, and sexually transmitted infections among post-deployment veterans who utilize digital social media for sexual-partner-seeking. Addictive Behaviors, 66, 96-100. doi:10.1016/j.addbeh.2016.11.015

Twohig, M. P., Crosby, J. M., \& Cox, J. M. (2009). Viewing Internet pornography: For whom is it problematic, how, and why? Sexual Addiction \& Compulsivity, 16(4), 253-266. doi:10.1080/10720160903300788

Walton, M. T., Cantor, J. M., Bhullar, N., \& Lykins, A. D. (2017). Hypersexuality: A critical review and introduction to the "sexhavior cycle". Archives of Sexual Behavior, 46(8), 2231-2251. doi:10.1007/s10508-017-0991-8

Weaver, J. B., Weaver, S. S., Mays, D., Hopkins, G. L., Kannenberg, W., \& McBride, D. (2011). Mental and physical health indicators and sexually explicit media use behavior by adults. Journal of Sexual Medicine, 8(3), 764-772. doi:10. 1111/j.1743-6109.2010.02030.x

Wéry, A., \& Billieux, J. (2015). Problematic cybersex: Conceptualization, assessment, and treatment. Addictive Behaviors, 64, 238-246. doi:10.1016/j.addbeh.2015.11.007

Wéry, A., \& Billieux, J. (2016). Online sexual activities: An exploratory study of problematic and non-problematic usage patterns in a sample of men. Computers in Human Behavior, 56, 257-266. doi:10.1016/j.chb.2015.11.046

Young, K. S. (2008). Internet sex addiction risk factors, stages of development, and treatment. American Behavioral Scientist, 52(1), 21-37. doi:10.1177/0002764208321339

Zakiniaeiz, Y., Cosgrove, K. P., Mazure, C. M., \& Potenza, M. N. (2017). Does telescoping exist in male and female gamblers? Does it matter? Frontiers in Psychology, 8, 1510. doi:10.3389/ fpsyg.2017.01510

Zhao, X., \& Cai, X. (2008). From self-enhancement to supporting censorship: The third-person effect process in the case of Internet pornography. Mass Communication and Society, 11(4), 437-462. doi:10.1080/15205430802071258

Zheng, L. J., Zhang, X., \& Feng, Y. (2017). The new avenue of online sexual activity in China: The smartphone. Computers in Human Behavior, 67, 190-195. doi:10.1016/j.chb.2016. 10.024

Zheng, L. J., \& Zheng, Y. (2014). Online sexual activity in mainland China: Relationship to sexual sensation seeking and sociosexuality. Computers in Human Behavior, 36, 323-329. doi:10.1016/j.chb.2014.03.062 